\title{
¿HAY UNA BURBUJA INMOBILIARIA EN BOGOTÁ? UN ESTUDIO POR SEGMENTOS DE MERCADO
}

\author{
Vanessa Cediel Sánchez* \\ Carlos Velásquez Vega**
}

Según el Dane, el índice de precios de vivienda nueva (IPVN) de Bogotá del segundo trimestre de 2014 superó en un $40 \%$ el promedio histórico y en un 15,4\% el cuarto trimestre de 1995, cuando alcanzó el nivel más alto anterior. La comparación de la evolución de este índice con el índice de costos de construcción de vivienda (ICCV) muestra que desde 2012 los precios de la vivienda crecen a tasas más altas. No obstante, el ICCV incluye costos de materiales, mano de obra, maquinaria y equipo, pero no el precio del suelo, y según datos de la Lonja, este mostró incrementos superiores al 30\% en términos reales en 2012 y 2013.

Este artículo examina más en detalle la evolución de los precios para determinar si los aumentos mencionados indican la existencia de una burbuja inmobiliaria. Aunque las burbujas se han estudiado en general desde el punto de vista teórico, y se han hecho variados estudios empíricos de casos específicos, no es fácil predecirlas para atenuar las consecuencias perjudiciales que provoca su estallido. En este artículo se define someramente qué es una burbuja inmobiliaria y se seleccionan los métodos estadísticos más apropiados para detectar su existencia, es decir, para determinar si las series de precios de vivienda nueva muestran o no un crecimiento explosivo. En el análisis econométrico se usan los datos de precios suministrados por

\footnotetext{
* Magíster en economía, investigadora de la Secretaría Distrital de Planeación de Bogotá, [vanessacediel@gmail.com].

** Magíster en economía, investigador de la Secretaría Distrital de Planeación de Bogotá, [alvelas50@gmail.com]. Agradecemos a José Gómez, Jair Ojeda y Natalia Sicard por sus valiosos aportes. Fecha de recepción: 20 de noviembre de 2014, fecha de modificación: 27 de febrero de 2015, fecha de aceptación: 29 de abril de 2015. Sugerencia de citación: Cediel S., V. y C. Velásquez V. “¿Hay una burbuja inmobiliaria en Bogotá? Un estudio por segmentos de mercado", Revista de Economia Institucional 17, 32, 2015, pp. 233-257. DOI: 10.18601/01245996.v17n32.08
} 
La Galería Inmobiliaria correspondientes al periodo enero de 2002mayo de 2014.

En la primera sección del artículo se revisa la literatura sobre burbujas inmobiliarias, en la segunda se describen los hechos estilizados del sector inmobiliario en Bogotá y en la tercera se exponen el método de análisis y las técnicas econométricas utilizadas. Después se presentan los resultados y por último se sintetizan las conclusiones del trabajo.

\section{REVISIÓN DE LA LITERATURA}

Los gobiernos suelen considerar que el sector de la construcción es estratégico porque proporciona un bien esencial para la población - la vivienda $-\mathrm{y}$, además, es intensivo en mano de obra y dinamiza la economía en su conjunto (Hofstetter et al., 2011; López y Salamanca, 2009). En el análisis del mercado inmobiliario y de las políticas públicas relacionadas hay que tener en cuenta que la vivienda, además de ser un activo que representa gran parte de la riqueza de los hogares, es un bien indivisible difícil de transar, con características y ubicación heterogéneas, altos costos de transacción y oferta cuasi inelástica ( $\mathrm{Sa}-$ lazar et al., 2012). Para entender el comportamiento de sus precios conviene aclarar que la vivienda es un activo y que el precio de los activos depende de la suma de sus rendimientos futuros, es decir, de las ganancias o pérdidas totales que obtiene el dueño en un periodo dado. La ganancia (pérdida) consta de dos elementos: la valorización (desvalorización) y la suma periódica derivada de su posesión.

En el mercado inmobiliario, los compradores de vivienda no suelen calcular el valor presente neto (precio observado) en función de los flujos futuros, y con respecto a los constructores y vendedores de vivienda se presentan asimetrías de información que pueden dar origen a fenómenos especulativos (Hwang y Smith, 2006).

Antes de revisar los estudios de burbujas inmobiliarias conviene aclarar en qué consisten. En términos simples, una burbuja, bien sea que se presente en el mercado bursátil o en el mercado de vivienda, es un aumento rápido y continuo de precios que no se explica por sus determinantes fundamentales sino por expectativas de aumentos adicionales y de rentabilidad creciente. Al inicio, un pequeño grupo de personas o empresas tiene expectativas de rentabilidad o valorización que lo llevan a invertir en un bien o activo, aun si su valor fundamental es bajo o 0 (Blanchard y Enrri, 2000); cuando llegan las ganancias, esas expectativas se difunden entre otros agentes que aumentan la inversión y, de ese modo, confirman la profecía del alza en precios, 
sin que esté basada en el comportamiento normal del mercado (Shi1ler, 2000). Una vez los precios llegan a un nivel insostenible, estos se derrumban y se generan pérdidas para los propietarios del bien o los inversionistas en el activo, que pueden llegar a afectar a las entidades crediticias que prestaron fondos para financiar la inversión.

No todas las burbujas especulativas son iguales, pues difieren según el tipo de activo y los factores que las desencadenan. De acuerdo, con Rosser y Gallegati (2012), que en esto siguen a Minsky y Kindleberger, existen tres tipos de burbujas según el tipo activo. El primero es el de las burbujas de bienes o materias primas, como la de los tulipanes en Holanda en 1636-1637, la de la plata en los ochenta y la del petróleo en 2007, en las que los precios aumentan más de lo que es posible dados sus determinantes fundamentales. El segundo es el de la inversión en activos que produce alzas y caídas de precios muy pronunciadas (p. ej., el auge y la crisis de las compañías de internet a finales de los noventa en Estados Unidos). El tercero es el de los mercados financieros, donde después de llegar a un pico de precios, estos caen en forma gradual y después se desploman produciendo estrés financiero y posibles riesgos sistémicos.

Las burbujas inmobiliarias pueden ser del segundo o del tercer tipo según esta clasificación. Por su parte, Crowe et al. (2011) las diferencian de acuerdo con los factores que las generan. Estos autores presentan un modelo P-VAR que usa datos de varios países en los que se ha observado este tipo de burbujas; sus resultados indican que las burbujas impulsadas por la expansión del crédito y el endeudamiento de los hogares tienen efectos macroeconómicos más pronunciados y duraderos sobre la economía (p. ej., una crisis) que las burbujas no ligadas al apalancamiento del sector financiero. Además, determinaron que, antes de la explosión de la burbuja, un aumento de la relación entre endeudamiento de los hogares y valor de la vivienda del 10\%, eleva en un $13 \%$ el precio nominal de la vivienda. Así mismo, advierten que, así la burbuja no sea consecuencia del endeudamiento excesivo, puede haber un colapso que desencadene una crisis moderada con algunas consecuencias: crecimiento de la construcción de vivienda inferior al potencial, restricción del crédito del sector financiero, aumento del desempleo y del déficit de vivienda, etc.

Puesto que las burbujas inmobiliarias pueden tener efectos graves o moderados en la economía y en la sociedad, es necesario detectarlas con toda la antelación posible y formular políticas preventivas. Las técnicas que más se usan para detectar burbujas son las pruebas univariadas de raíz unitaria de cola larga, que diferencian entre la no 
estacionariedad de una serie y su carácter explosivo, y las pruebas de cointegración que determinan los equilibrios de precios de largo plazo (Gómez et al., 2013).

Phillips et al. (2011) muestran que es muy complejo detectar las burbujas de activos debido a que tienen una estructura no lineal y, por tanto, que se necesita desarrollar métodos novedosos para detectar los incrementos exuberantes de precios. Ellos utilizan una prueba de Dickey-Fuller de cola larga recurrente y la comparan con una prueba recurrente hacia atrás para detectar burbujas del índice S\&P 500, con datos del periodo enero de 1871-diciembre de 2010. Con ese método lograron identificar los fenómenos especulativos asociados con el colapso de 1930, el boom de la posguerra de 1954, el viernes negro de 1987, la burbuja de las empresas de internet entre 1995-2000 y la crisis financiera de 2008.

En Colombia, Gómez et al. (2013) emplearon el método de Phillips et al. para detectar burbujas inmobiliarias usando el índice de precios de vivienda nueva deflactado por el IPC y por el IPC de arrendamientos. El resultado con el índice deflactado por el IPC es que hay evidencia de la aparición de burbujas con el 95\% de confianza, entre ellas una a finales de los noventa y otra iniciada en junio de 2012. Los resultados son similares cuando se usa el índice deflactado por el IPC de arrendamientos.

Las pruebas mencionadas solo se basan en el crecimiento de los precios y son más robustas a medida que se tienen series de tiempo más largas. Al no tener en cuentan los factores que explican el aumento de precios no se puede determinar si el alza responde a los fundamentales o a la especulación. Por ello, estas pruebas no permiten determinar nítidamente si existe una burbuja, aunque dan indicios de que se puede estar gestando una.

A diferencia de las técnicas univariadas, el análisis multivariado detecta el desajuste entre los precios observados y los precios determinados por los fundamentales. En estos análisis, que emplean series de tiempo y modelos estructurales, la oferta y la demanda se relacionan en forma conjunta y simultánea. Clavijo et al. (2005) caracterizan los determinantes socioeconómicos de la oferta y la demanda mediante un modelo de ecuaciones simultáneas, y encuentran que el precio de la vivienda aumenta cuando crecen los precios de la construcción, la cantidad ofrecida y la tasa de interés de referencia, el DTF. Además, que la demanda aumenta conforme crecen la riqueza financiera y el ingreso, y que disminuye cuando se elevan los precios de la vivienda y la tasa de desempleo. 
Joshi (2006) analiza el mercado inmobiliario de India usando un modelo de vectores autorregresivos estructurales (SVAR) que determina el desajuste entre el precio observado y el precio de equilibrio provocado por la tasa de interés, los desembolsos de crédito no alimentario (proxy del crédito hipotecario) y el PIB. Sus resultados indican que si bien no se presentó un desajuste de precios, la tasa de interés y los desembolsos influían más en los precios que el PIB, de modo que hay que observar cuidadosamente el comportamiento de estas variables y la evolución de dicho mercado.

Siguiendo a Joshi, Salazar et al. (2012) usan el SVAR para calcular el peso de los fundamentales en el precio de la vivienda en Colombia y determinar si hay un desajuste entre el precio calculado y el observado. Sus resultados sugieren que no hay desajuste y que el aumento de los precios de la vivienda se debe ante todo al encarecimiento del precio del suelo y al aumento de la demanda, no a la tasa de interés de referencia ni a los desembolsos. Para complementar los resultados, emplearon dos modelos de vectores de corrección de errores (VEC), de los precios de oferta y demanda, para encontrar los precios de equilibrio de largo plazo y evaluar el comportamiento simultáneo de la oferta y la demanda. Los resultados correspondientes constatan que no hay desajuste entre el precio observado y los precios estimados, y sugieren que los precios de oferta en el largo plazo aumentan cuando se incrementan las tasas de inversión en construcción, los costos de la construcción y los precios del suelo, mientras que disminuyen cuando aumenta el stock de vivienda y aumentan con un mayor costo de uso de la vivienda. En el corto plazo, el precio de demanda aumenta con la mayor riqueza financiera.

Si bien los análisis multivariados, en especial los análisis estructurales, permiten establecer las relaciones entre el precio de la vivienda y los fundamentales, se debe señalar que, igual que las pruebas univariadas, tienen limitada capacidad predictiva pues no han detectado los colapsos de las burbujas, como demostró Evans (1991). Además, exigen imponer restricciones y supuestos fuertes cuando se calculan las elasticidades, en particular los modelos SVAR o SVEC.

\section{HECHOS ESTILIZADOS}

\section{Precios de LA VIVIENDA, 1988-2014}

La gráfica 1 muestra el IPVU de Colombia calculado por el Banco de la República (BR) para el periodo 1988I-2014I; el IPVN de Bogotá reportado por el Dane para el periodo 2000I-2014II; el índice 
de precios de vivienda (IPV) de Bogotá reportado por el DNP para el periodo 1994I-2014II ${ }^{1}$; y el índice de precios de vivienda nueva (IPVNGALERIA) de Bogotá ${ }^{2}$, calculado por los autores con datos de La Galería Inmobiliaria, para el periodo 2005I-2014II. Todos los índices se deflactaron con el IPC.

La serie más larga muestra que en el cuarto trimestre de 1995 (1995IV) los precios de la vivienda alcanzaron el punto más alto anterior, pero como resultado del colapso del sistema UPAC, en el tercer trimestre de 1996 el precio se desplomó y cayó hasta el primer trimestre de 2004, cuando empezó la recuperación asociada a factores como el crecimiento del ingreso de los hogares, entre otros (Piraquive y Hernández, 2014). Desde esa fecha los precios han aumentado hasta tal punto que a comienzos de 2011 llegaron a otro alto nivel histórico, y siguen creciendo. Según esta serie del BR, en el primer trimestre de 2014 el IPVU fue un 24,3\% mayor que en el cuarto trimestre de 1995, uno de los niveles más altos desde que se tiene información de precios. Y, con respecto al promedio histórico (74,8 en 1988I-2014I), los precios del primer trimestre de 2014 son un $40 \%$ mayores.

Gráfica 1

Índices de precios de la vivienda, 1988I-2014II

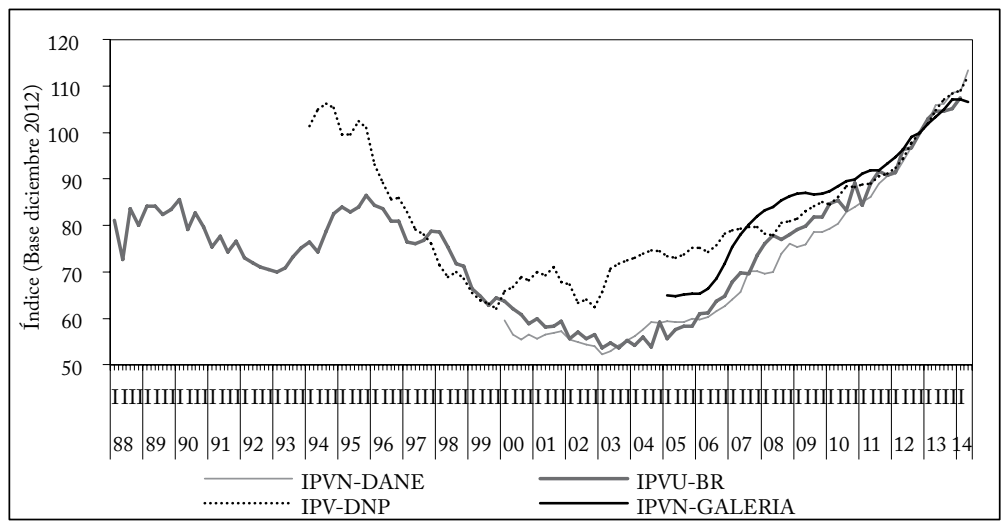

Fuente: IPVN: Dane, IPVU: BR, IPV: DNP, IPVN: Galería Inmobiliaria; cálculos propios.

${ }^{1}$ E1 IPV es mensual. Para presentar en la misma gráfica esta serie junto con las demás, la serie del IPV se trimestralizó tomando el dato del último mes del trimestre.

${ }^{2}$ La Galería Inmobiliaria reporta mensualmente los precios de venta de vivienda nueva sobre planos. Para calcular el índice se tomó el promedio trimestral del precio del metro cuadrado y luego se transformó en índice tomando un año de base; después se calculó el promedio anual. 
En un trabajo reciente, Jaramillo y Cuervo, con datos de precios de la vivienda usada en Bogotá, examinan la desviación de los precios con respecto a la tendencia y encuentran que en "la presente coyuntura [se] alcanza la mayor desviación hacia arriba de los últimos cuarenta años, superando esta tendencia en un $65 \%$. Estas si son cifras que hacen pensar en una burbuja especulativa" (2014, 36-37). Estos análisis de los precios han despertado la preocupación de algunos analistas por la posible existencia de una burbuja inmobiliaria. No obstante, las opiniones están divididas.

Algunos analistas consideran que no hay burbuja y argumentan que el aumento de precios se debe a uno de sus principales fundamentales -el precio del suelo-, como Salazar et al. (2012). A este respecto cabe señalar que esto olvida que el alza de las expectativas de valorización de las viviendas es la que provoca el aumento de los precios del suelo, el cual luego se traduce en mayores precios de la vivienda. Salazar et al. no consideran esta retroalimentación, pues en su modelo SVAR adoptan el supuesto fuerte de que los precios de la vivienda no afectan el precio del suelo. Además, el argumento que desvirtúa la existencia de burbujas inmobiliaria con base en el comportamiento de los precios de suelo soslaya el hecho de que puede haber especulación en el precio de este componente.

A diferencia de Salazar et al., Jaramillo y Cuervo (2014, 40), que analizan los precios inmobiliarios, calculan la desviación porcentual del precio promedio real de la vivienda usada y del precio real del suelo frente a cada una de sus respectivas tendencias, y señalan que en "la fase de crecimiento actual, la desviación positiva del precio de los inmuebles sobre su tendencia es anterior y más acentuada que la del precio de los terrenos. Esto haría pensar que, contra ciertas percepciones, en la coyuntura actual no parece ser el precio del suelo el que esté liderando la inflación de precios, sino al contrario. De hecho la desviación sobre su tendencia en el caso del precio del espacio construido supera netamente el punto más alto del ciclo ascendente anterior, mientras que en el caso del precio del suelo todavía no alcanza su nivel" (ver la gráfica 2).

Además del argumento basado en los precios del suelo, la existencia de una burbuja suele rechazarse con otros dos argumentos: el bajo nivel del inventario terminado en manos de los constructores y los buenos indicadores de calidad de la cartera hipotecaria. Con respecto al primero, los datos de La Galería Inmobiliaria muestran, en efecto, que en junio de 2014 la oferta terminada representaba apenas el 2,3\% del total (Bogotá y 10 municipios de la Sabana). Esa cifra no invalida 
la existencia de una burbuja, pues nada dice respecto de si hay o no desajuste de precios. E1 bajo porcentaje de vivienda terminada es una consecuencia del actual sistema de ventas sobre planos, en el que solo se inicia la construcción si se ha garantizado el éxito del proyecto, es decir, si ya se ha vendido; así, la oferta terminada no queda en manos de los constructores sino de los compradores.

Gráfica 2

Desviación porcentual de los precios con respecto a la tendencia Promedio del suelo y de la vivienda 1970-2012

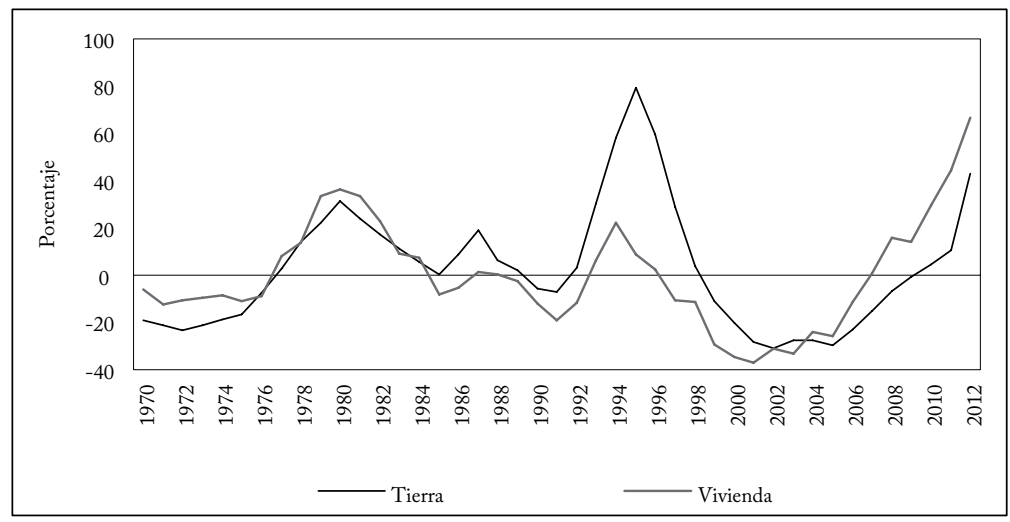

Fuente: Jaramillo y Cuervo (2014).

El segundo argumento se basa en los indicadores de cartera, que hoy reflejan una cartera hipotecaria sana. $\mathrm{E} 1$ indicador de mora (cartera vencida/ cartera bruta) llegó a niveles de más del 20\% entre 1999 y 2003. Desde 2004 la proporción de cartera vencida empezó a descender hasta llegar a menos del $4 \%$. Un comportamiento similar se observa en Bogotá (gráfica 3).

La cartera hipotecaria como proporción del PIB indica que los hogares hoy usan menos crédito para financiar la compra de vivienda. Esa proporción hoy está en un nivel inferior a los observados en la crisis de finales de los noventa. Entre 1998 y 1999 llegó al 10\%; si bien desde 2011 muestra una tendencia creciente, hoy no llega al 5\% (gráfica 4). A los bajos niveles de cartera hipotecaria/PIB y a los buenos indicadores de cartera se suma el bajo endeudamiento de los hogares. La relación entre el préstamo y el valor de la viviendas que no son viviendas de interés social (VIS) ha girado alrededor del 60\% (entre 2007 y 2012) y la de VIS, de alrededor del 50\%, inferior a la observada en 1997 (70\% y 60\%, respectivamente) y a los promedios internacionales (BR, 2013; Crowe et al., 2011). 
Gráfica 3

Calidad de la cartera hipotecaria, 1995II-2014II

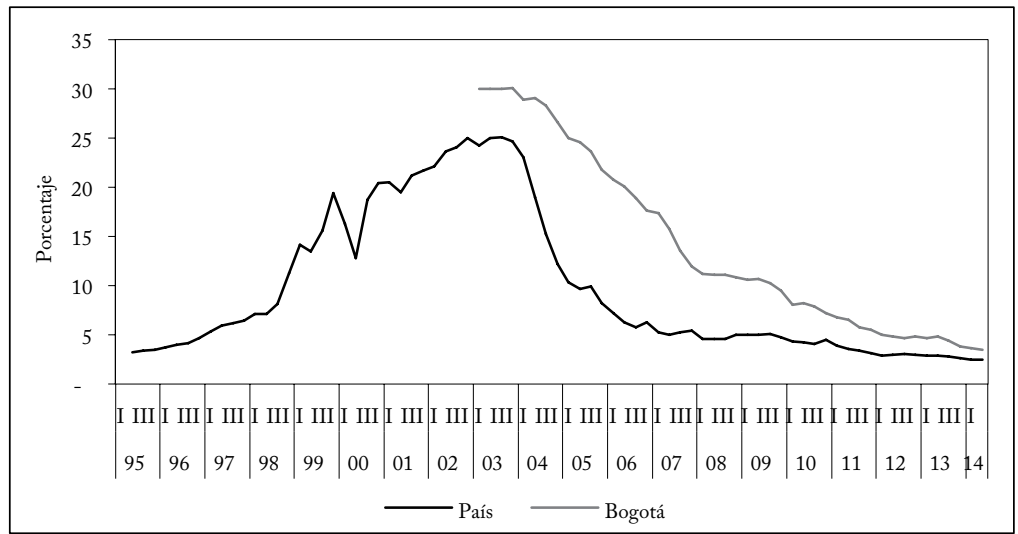

Fuente: cartera país: Superintendencia Financiera, Cartera Bogotá: Dane; cálculos propios.

Gráfica 4

Cartera hipotecaria/PIB, 1996II -2014II

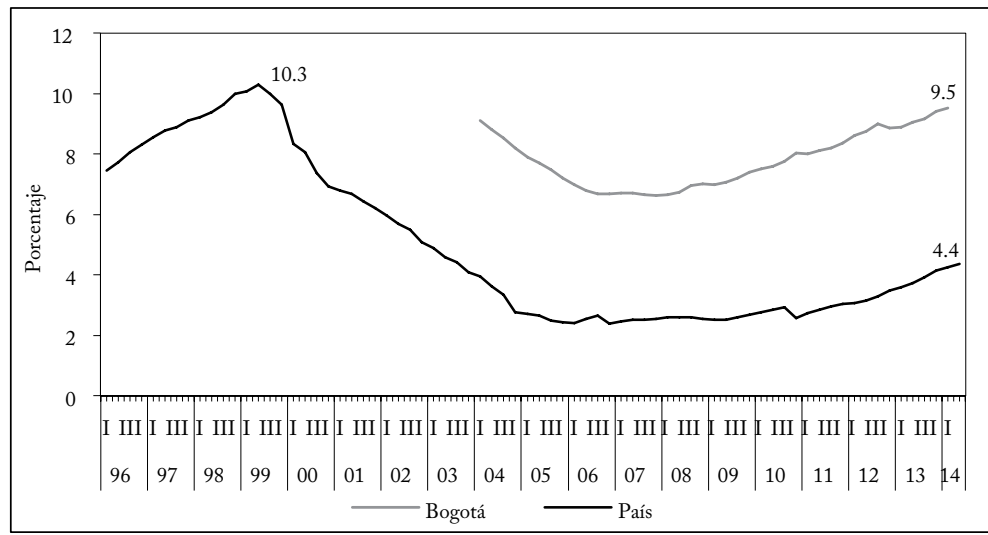

Fuente: cartera país: Superintendencia Financiera, cartera Bogotá: Dane; cálculos propios.

Si bien la calidad de la cartera y los niveles de cartera hipotecaria como proporción del PIB podrían disipar el temor de que haya una burbuja inmobiliaria financiada con crédito, se debe señalar que la cartera hipotecaria viene creciendo en forma significativa, en términos absolutos y relativos, en particular desde 2011. En el segundo trimestre de 2014, el saldo ascendió a 23 billones de pesos de 2005 (gráfica 5), el 86\% del saldo más alto observado en el cuarto trimestre de 1998. La evolución de la cartera a nivel nacional en términos porcentuales muestra que desde comienzos de 2011 la cartera hipotecaria viene creciendo a una tasa promedio anual de un 20\% (gráfica 6). En Bogotá se observa un comportamiento similar. 


\section{Gráfica 5}

Cartera hipotecaria, país y Bogotá; 1995II-2014II

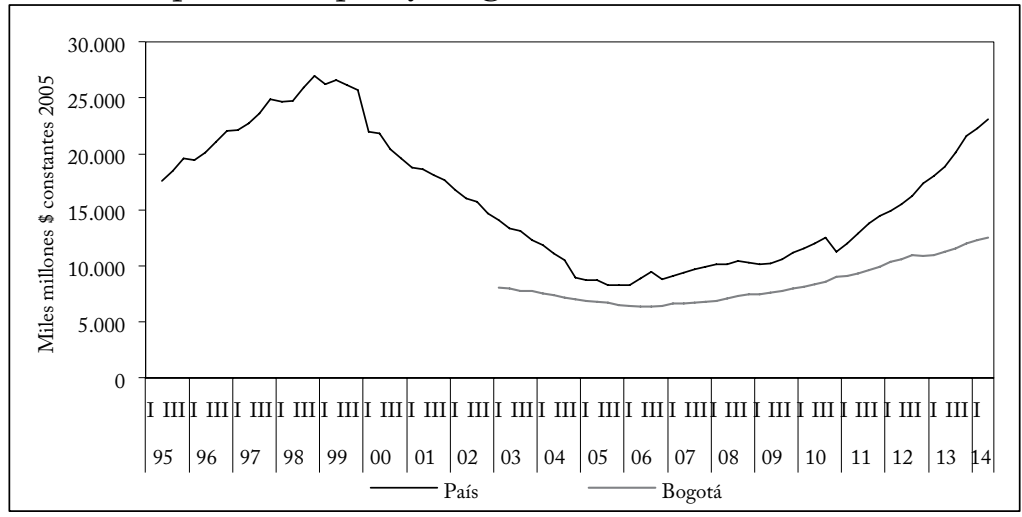

Nota: deflactado por el IPC.

Fuente: cartera país: Superintendencia Financiera, cartera Bogotá: Dane; cálculos propios.

\section{Gráfica 6}

Crecimiento real de la cartera hipotecaria, país y Bogotá; 1996II-2014II

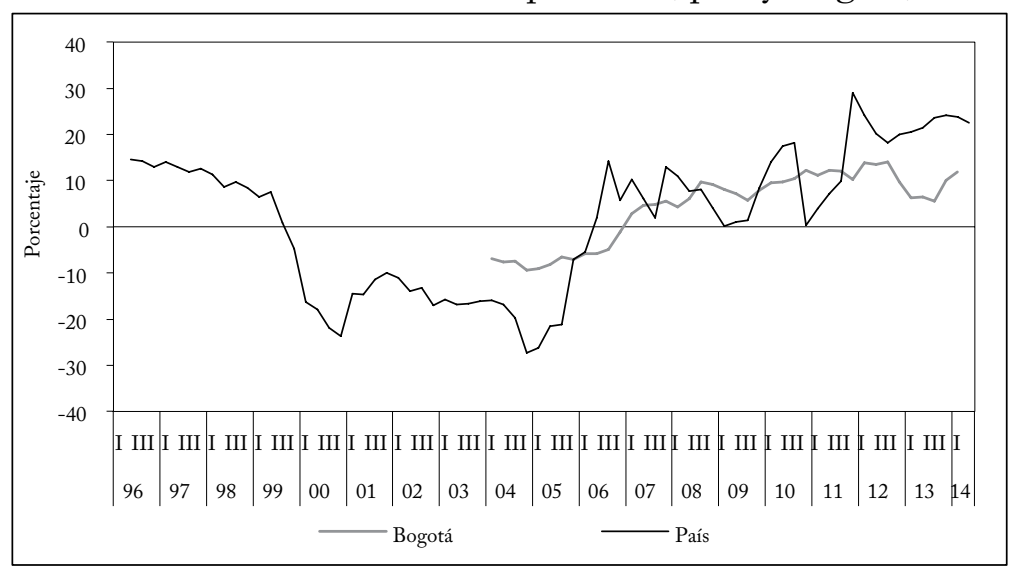

Nota: deflactado por el IPC.

Fuente: cartera país: Superintendencia Financiera, cartera Bogotá: Dane; cálculos propios.

También se argumenta que si bien los altos precios de la vivienda son similares e incluso mayores que los de los años noventa, las condiciones actuales son diferentes, ya que en esos años hubo un desequilibrio que creó las condiciones propicias para el colapso de finales de esa década: aumento de las tasas de interés y caída del ingreso de los asalariados, mientras que hoy los altos precios de la vivienda obedecen a la presión de la demanda de los hogares, que han experimentado un aumento sus salarios, y a las tasas de interés, que hoy son las más 
bajas de la historia. No obstante, como ya se señaló, la formación de una burbuja inmobiliaria no depende del tipo de financiación. Conforme a la descripción anterior, no hay una burbuja inducida por la expansión del crédito, aunque se debe hacer seguimiento continuo del comportamiento de la cartera hipotecaria. La explosión de una burbuja financiada con apalancamiento tiene mayores efectos negativos sobre la economía que una burbuja financiada con recursos propios, pero no se debe olvidar que un desplome de precios tendría graves consecuencias, como el deterioro de la riqueza de los hogares, el freno de la construcción - un sector que dinamizador el desempeño económico del país-, el aumento del desempleo y otras más.

\section{SEgMENTACIÓN DEL MERCADO}

Para entender la evolución de los precios de la vivienda no basta analizar el índice total de la ciudad, pues este omite las diferencias de precios por estrato. Las características de la oferta y de la demanda de vivienda difieren según el segmento del mercado. Por el lado de la oferta, las diferencias se reflejan ante todo en el tipo de bien que se ofrece y en la rentabilidad para los constructores. Por el lado de la demanda, obedecen básicamente a dos razones: la capacidad de pago de los hogares y las necesidades de cada tipo de hogar (SDP, 2014a).

En la gráfica 7 se presenta el índice de precios de la vivienda nueva y usada para tres segmentos de la población bogotana, bajo, medio y alto, según las cifras de Jaramillo y Cuervo (2014) y del DNP. A primera vista se observa que en términos generales el comportamiento de las tres series es similar. Pero si se observan con mayor detalle, el segmento que pone la pauta en el comportamiento de los precios es el estrato alto. Como dicen Jaramillo y Cuervo, "se mantiene la pauta de que los precios comienzan a crecer un poco antes en el estrato de ingresos elevados".

La mayor rentabilidad que se puede obtener por la venta de viviendas para estratos altos en comparación con la venta de vivienda para estratos bajos ha llevado a que las empresas concentren su actividad en el primer segmento. Así lo muestra la gráfica 8, que presenta las ventas de vivienda nueva por rango de precios: no VIS, VIS y VIP, en el acumulado de doce meses, un punto que ya antes habían señalado Cuervo y Jaramillo (2009). 


\section{Gráfica 7}

Precios de la vivienda por segmento

Vivienda usada

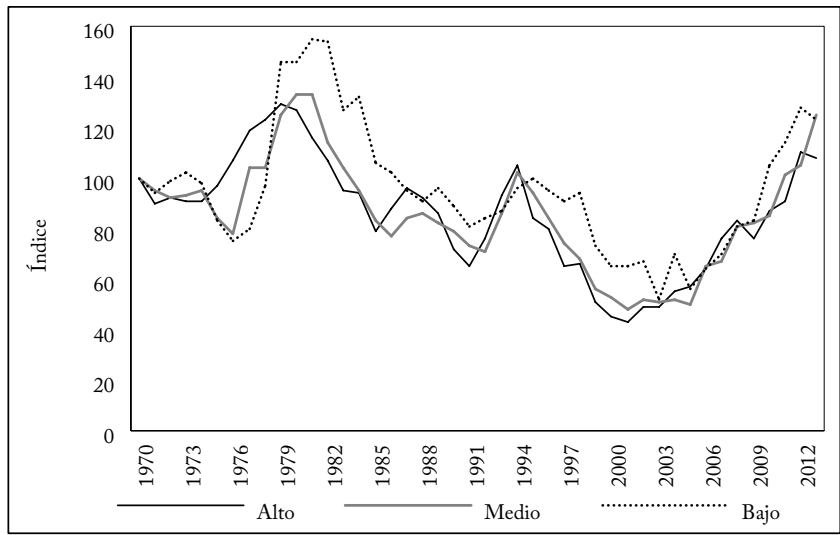

Fuente: Jaramillo y Cuervo (2014)

Vivienda nueva

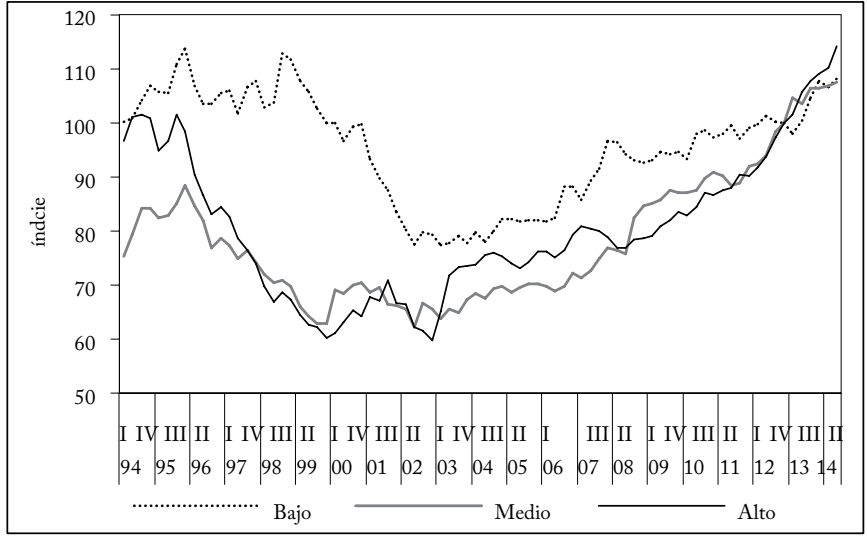

Fuente: DNP, cálculos propios.

Otro factor que genera diferencias en los segmentos del mercado por estrato socioeconómico es el diferencial entre ventas de vivienda y formación de hogares, el cual es además un indicativo del nivel de oferta que debería existir en la ciudad para satisfacer las necesidades de los hogares.

La gráfica 9 muestra las cifras de ventas de vivienda nueva y de formación de nuevos hogares. Según el Dane, en Bogotá se forman anualmente cerca de 60 mil hogares y según La Galería Inmobiliaria se venden anualmente cerca de 30 mil viviendas nuevas, es decir, apenas la mitad de las que requieren los nuevos hogares. 
Gráfica 8

Ventas de vivienda nueva por rango de precios

Acumulado 12 meses, enero de 2006-junio de 2014

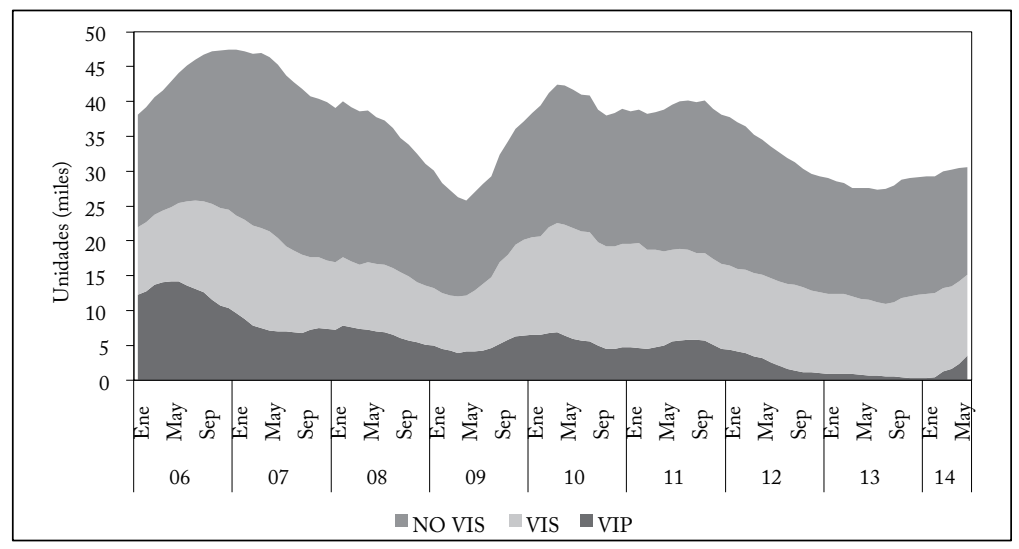

Fuente: La Galería Inmobiliaria, cálculos propios.

Cuando se observa esta diferencia por estratos, se aprecia un desfase en los estratos 2 y 3 . Pese al visible aumento de la venta de vivienda en estrato 2 en la primera parte de 2014, aún es insuficiente para suplir las necesidades de los nuevos hogares pertenecientes a este estrato (gráfica 9a); igual sucede en el estrato 3 (gráfica 9b). En cambio, desde que se dispone de esta información en los estratos 4, 5 y 6 las ventas son visiblemente mayores que la formación de nuevos hogares (gráficas 9c, 9d y 9e), aunque la tendencia ha empezado a revertirse.

Gráfica 9

Ventas de vivienda nueva vs formación de hogares

Acumulado doce meses, enero de 2003-agosto de 2014

a. Estrato 2

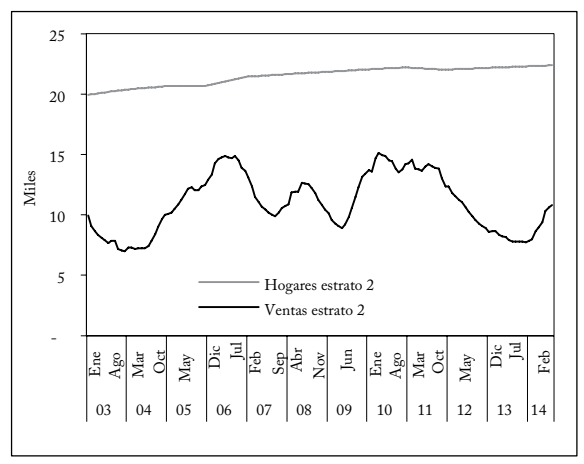

b. Estrato 3

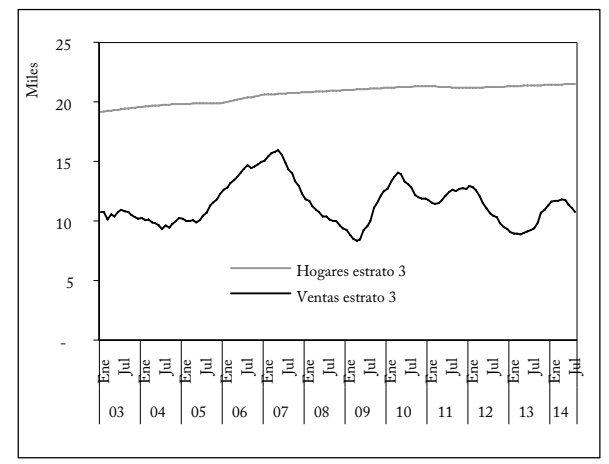


c. Estrato 4

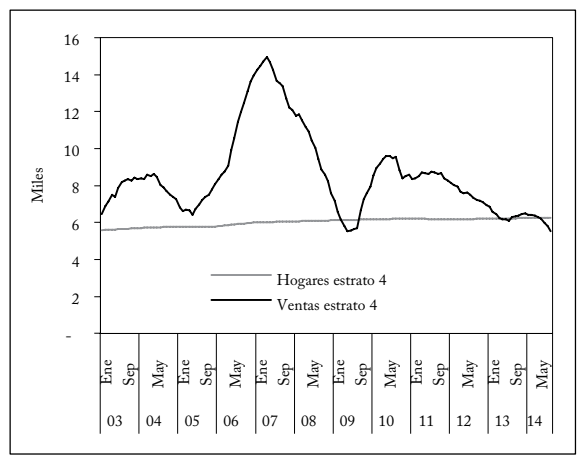

d. Estrato 5

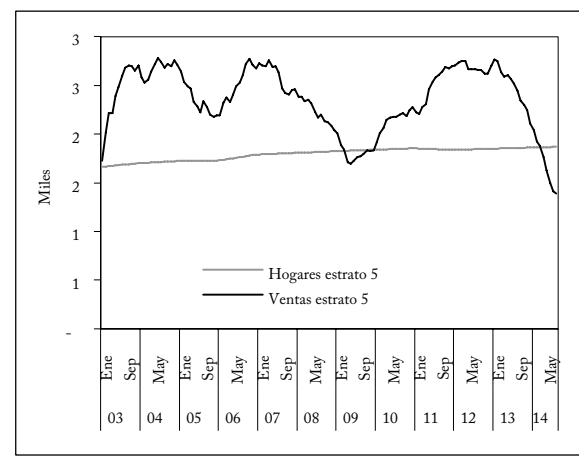

e. Estrato 6

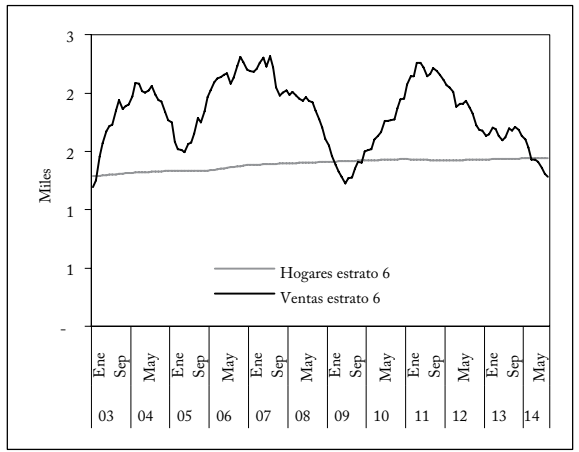

Fuente: ventas: La Galería Inmobiliaria, nuevos hogares: Dane; cálculos propios.

Cuando las ventas de vivienda nueva caen es previsible que en los años siguientes aumente la oferta de vivienda usada; así se evidencia en los estratos 5 y 6 (ver el anexo). Y esto indica que se está en la parte del ciclo de ajuste entre ventas de vivienda nueva y vivienda usada, pues la reducción de la venta de unidades nuevas permite colocar y depurar el stock de vivienda usada. Una prueba de que se venden más viviendas de las que se requieren en los estratos 5 y 6 es que allí no hay déficit cuantitativo, y una prueba de que las ventas de vivienda nueva no suplen las necesidades de los hogares de bajos ingresos es el déficit en los estratos 1, 2 y 3 (cuadro 1). El desfase entre ventas y formación de hogares en los estratos bajos indica que la venta de vivienda nueva no solo no satisface las necesidades de los nuevos hogares de estos estratos sino que es insuficiente para reducir el déficit de vivienda.

Otro indicador relevante en el mercado de vivienda es el de rotación, que se presenta en la gráfica 10 y que mide el número de meses que tardaría en venderse la oferta disponible, dadas las ventas 
del trimestre. Como se puede apreciar, cuanto más bajo es el estrato menor es el indicador. La mayor rotación en los estratos bajos frente a los altos indica una oferta limitada y una demanda muy dinámica. Para las viviendas de estratos 3, 4, 5 y 6, el tiempo que tarda la venta tiende a aumentar, sobre todo en los estratos 5 y 6 . No solo se requiere más tiempo para vender nuevas viviendas en los estratos 4,5 y 6, también se tarda cada vez más tiempo para arrendar vivienda en estos estratos (gráfica 11).

Cuadro 1

Déficit de vivienda, 2011

\begin{tabular}{lrrrrrrr}
\hline \multirow{2}{*}{ Estrato } & \multicolumn{1}{c}{ Total } & \multicolumn{2}{c}{ Hogares en déficit } & \multicolumn{2}{c}{ Déficit cuantitativo } & \multicolumn{2}{c}{ Déficit cualitativo } \\
\cline { 2 - 7 } & \multicolumn{1}{c}{ Hogares } & Hogares & \multicolumn{1}{c}{$\%$} & Hogares & \multicolumn{1}{c}{$\%$} & Hogares & \multicolumn{1}{c}{$\%$} \\
\hline Estrato 1 & 172.199 & 47.946 & 27,8 & 27.044 & 15,7 & 20.902 & 12,1 \\
Estrato 2 & 830.377 & 140.747 & 16,9 & 63.381 & 7,6 & 77.366 & 9,3 \\
Estrato 3 & 798.621 & 58.933 & 7,4 & 22.805 & 2,9 & 36.128 & 4,5 \\
Estrato 4 & 232.491 & 3.909 & 1,7 & 581 & 0,2 & 3.328 & 1,4 \\
Estrato 5 & 69.360 & 1.176 & 1,7 & 0 & 0,0 & 1.176 & 1,7 \\
Estrato 6 & 53.594 & 605 & 1,1 & 0 & 0,0 & 605 & 1,1 \\
Sin información & 29.231 & 4.730 & 16,2 & 2.718 & 9,3 & 2.012 & 6,9 \\
Total Bogotá & 2.185 .874 & 258.046 & 11,8 & 116.529 & 5.3 & 141.517 & 6,5 \\
\hline
\end{tabular}

Fuente: EMB (2011), cálculos propios.

La baja rotación y la tendencia decreciente en el estrato 2 reflejan una demanda insatisfecha. Los altos y crecientes niveles de rotación en los estratos 4, 5 y 6 muestran que en estos segmentos del mercado las ventas responden más a necesidades de inversión que de vivienda, sobre todo en los estratos 5 y 6 , donde no hay déficit cuantitativo de vivienda.

Gráfica 10

Indicador de rotación por estrato, Bogotá, enero de 2002-agosto de 2014

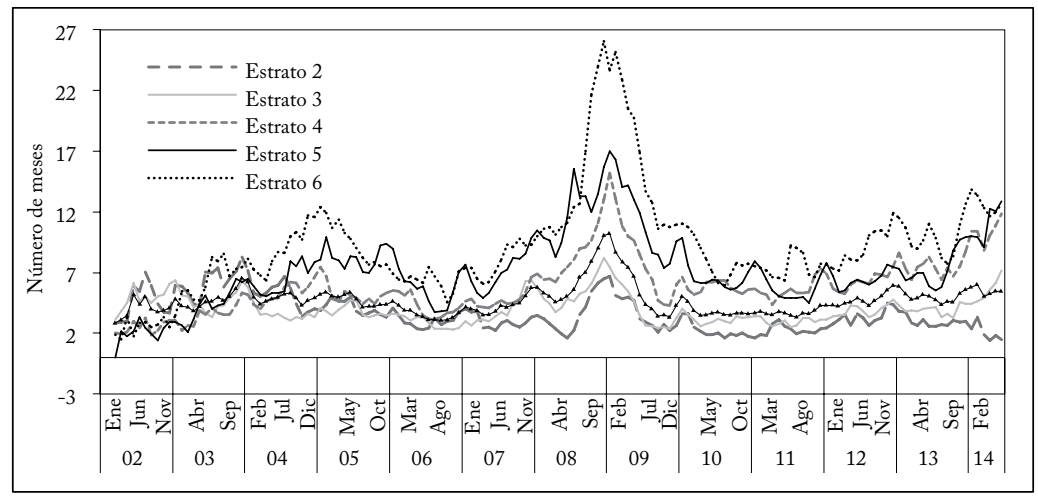

Fuente: La Galería Inmobiliaria, cálculos propios. 
La Galería Inmobiliaria suministra información del precio de venta de vivienda nueva por zonas, y se puede calcular el precio por metro cuadrado en las zonas en que divide la ciudad: Chicó, Multicentro, Centro, Salitre, Norte, Modelia, Suba, Occidente y Suroriente. E1 mapa 1 presenta esta delimitación ${ }^{3}$; las gráficas de pastel indican la distribución de las ventas por estratos en cada zona.

\section{Gráfica 11}

Tiempo de arriendo, enero de 2008-agosto de 2014

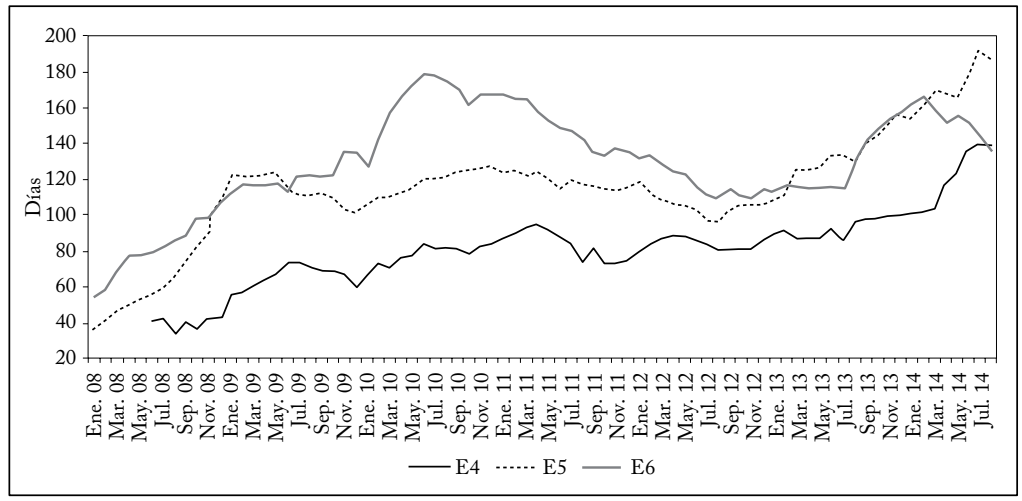

Fuente: La Galería Inmobiliaria.

Gráfica 12

Valor del metro cuadrado por zona, Bogotá; 2004I-2014II

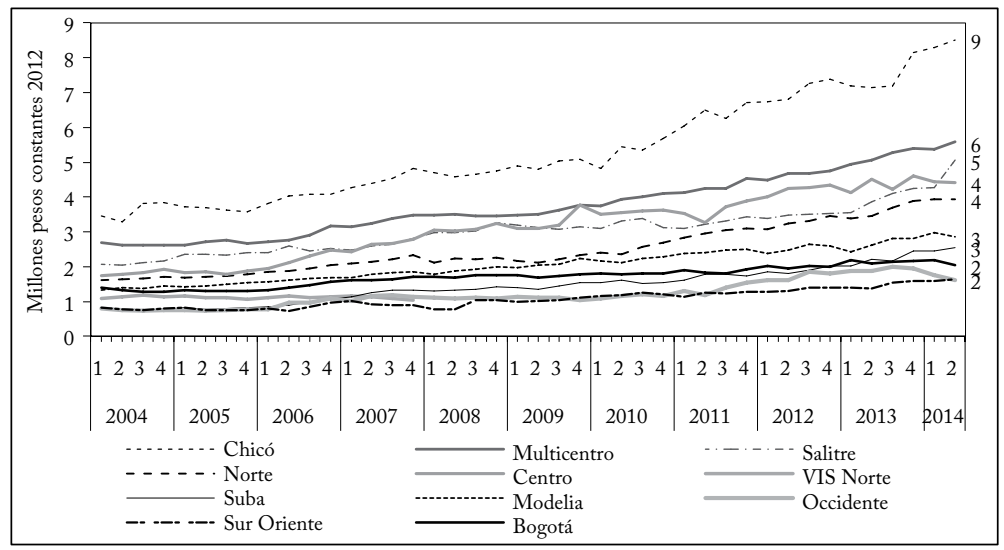

Fuente: La Galería Inmobiliaria, cálculos propios.

La gráfica 12 muestra el valor por metro cuadrado de la vivienda nueva en Bogotá y en las nueve zonas. Es claro que los precios difieren según la zona. En el sur de la ciudad, en las zonas del suroriente

3 Esta delimitación responde a las necesidades de la firma. 
y el occidente el precio por metro cuadrado es de unos 2 millones de pesos, mientras que en el norte, en las zonas de Multicentro y Chicó, llega a 6 y 9 millones de pesos respectivamente. No es extraño que en esas zonas se concentren las viviendas de estrato más alto y los hogares de mayores ingresos.

De los hechos estilizados que acabamos de presentar se puede concluir que además de las diferencias en los niveles de precios, los factores que determinan el precio de la vivienda difieren entre estratos, por lo cual se requiere un análisis desagregado.

\section{Mapa 1}

Zonas definidas por La Galería Inmobiliaria, mayo de 2012-abril de 2013

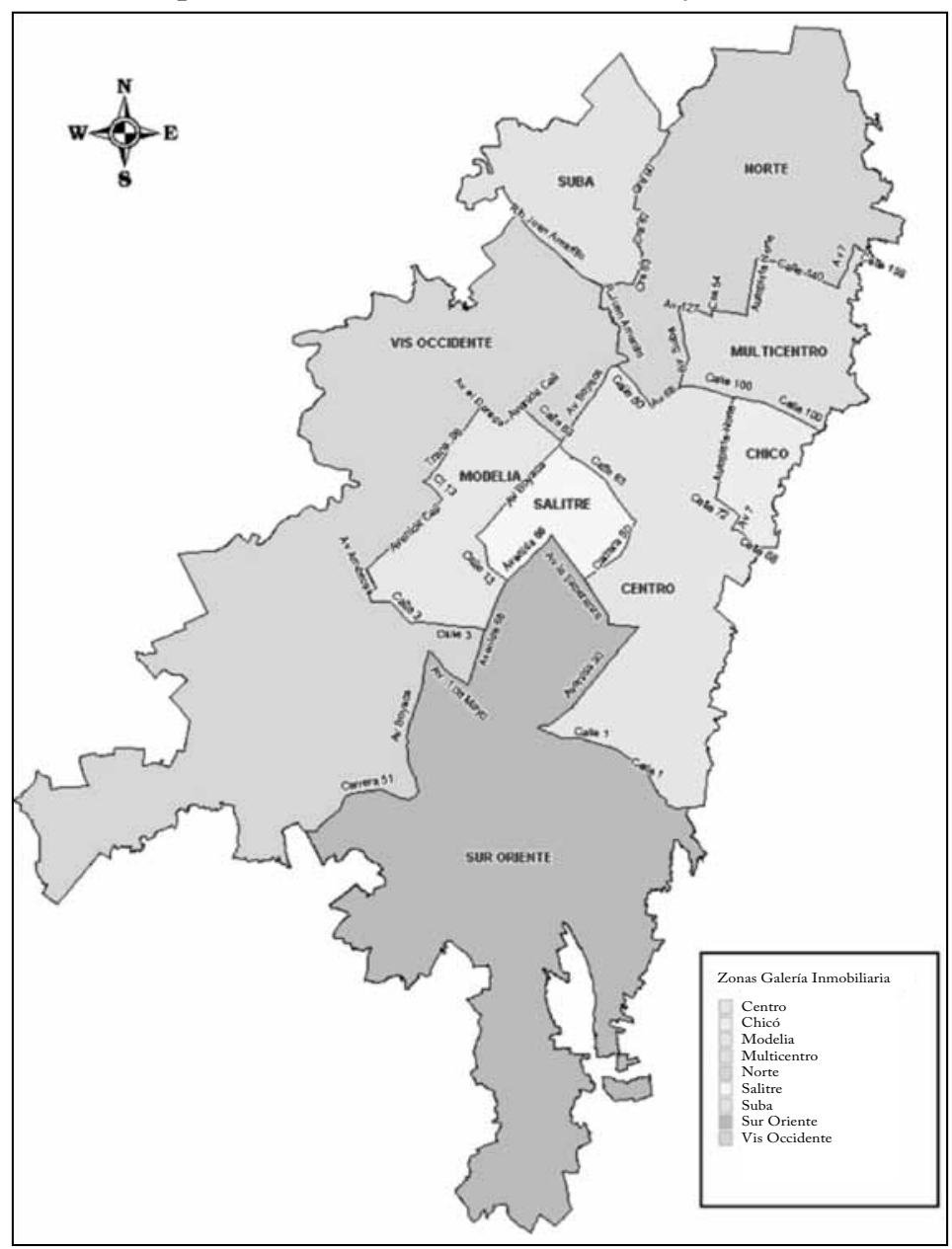

Fuente: La Galería Inmobiliaria, DICE-SDP. 


\section{METODOLOGÍA}

En la literatura se usan diversos métodos estadísticos para analizar la existencia de burbujas de precios; el uso de uno u otro depende del criterio del investigador y de lo que entiende como burbuja. Aquí se emplea un método univariado dadas las limitaciones de las técnicas multivariadas antes mencionadas, como la adopción de supuestos fuertes para hacer las estimaciones. Recientemente se ha empezado a usar métodos basados en pruebas de raíz unitaria, de las cuales ya hay varias versiones, entre ellas las pruebas de Dickey-Fuller aumentada (ADF), supADF (SADF), backwardsupADF (BSADF) y generalizedsupADF (GSADF). Estas pruebas de raíz unitaria forman parte de las técnicas de series de tiempo univariadas y su objetivo es determinar si una serie de tiempo muestra un comportamiento explosivo.

En la prueba de Dickey-Fuller aumentada (ADF) se estima la siguiente regresión, usando mínimos cuadrados ordinarios (MCO):

$\Delta y_{t}=c+\beta t+\varphi y_{t-1}+\sum_{i=1}^{p} \gamma_{i} \Delta y_{t-1}+\varepsilon_{t}$

donde la variable dependiente, $\Delta y_{t}$, es la primera diferencia de la serie que se desea analizar y la variable independiente, $y_{t-1}$, es la serie rezagada. Se puede incluir, además, una constante, $c$, y una pendiente determinística, $t$. Para que el modelo sea confiable, el término de error, $\varepsilon_{t}$, debe ser de ruido blanco, es decir, debe tener una distribución normal, varianza constante y los errores no deben estar autocorrelacionados. En caso de presentar problemas de correlación se pueden resolver incluyendo la primera diferencia de la serie rezagada $p$ periodos, $\Sigma_{i=1}^{p} \gamma_{i} \Delta y_{t-1}$.

El estadístico ADF corresponde al coeficiente de la variable independiente. Para determinar si la serie es o no estacionaria se plantea la hipótesis nula $H_{0}: \varphi=0$, esto es, que sea no estacionaria, frente a la hipótesis alternativa $H_{0}: \varphi \neq 0$. Esta última implica que $\varphi$ puede ser menor o mayor que 0 . En el primer caso sería estacionaria y en el segundo, explosiva. Puesto que la hipótesis alternativa es bilateral, hay dos regiones de rechazo, de modo que si el estadístico cae en la zona de rechazo del lado izquierdo, la serie es estacionaria, y si cae en la zona de rechazo del lado derecho es explosiva. Una vez se calcula el valor del estadístico, se compara con los valores críticos para determinar el comportamiento de la serie.

La prueba ADF permite identificar si la serie es explosiva o no. Sin embargo, el objetivo va más allá: determinar si en la serie analizada hay episodios de crecimiento explosivo; para ello se utiliza la prueba $\mathrm{SADF}$, la cual es útil si no hay más de un episodio explosivo. En este 
caso, la hipótesis nula es que no hay crecimiento explosivo y la alternativa que sí lo hay.

La prueba BSADF es más rigurosa porque puede identificar la existencia de varios episodios de crecimiento explosivo. En este caso la hipótesis nula es la inexistencia de periodos explosivos y la alternativa es que se observa al menos un periodo. Una vez se encuentra crecimiento explosivo en las series empleando la prueba anterior, se procede a determinar desde cuándo se presenta el crecimiento explosivo de los datos.

En este artículo se siguen los trabajos de Phillips et al. (2011) y Gómez et al. (2011) que, aplicando pruebas de Dickey-Fuller aumentadas de cola larga recurrentes hacia adelante (SADF) y hacia atrás (BSADF), buscan que en un periodo mínimo (no menor de 12 meses) se observe un comportamiento explosivo de los precios de la vivienda, bien sea de alza o caída que indique la presencia o explosión de una burbuja.

\section{RESULTADOS}

Los datos que se usan para hacer las pruebas provienen de La Galería Inmobiliaria, que reporta los datos de precios de venta de vivienda nueva sobre planos, con periodicidad mensual, desde 2003. Esta serie es muy corta, pues solo cubre la última década. Puesto que las series incluyen un componente irregular, ocasionado por factores de corto plazo o imprevistos, se deben suavizar para aislar ese componente y para evitar sesgos de estacionalidad. A partir del IPVNG se estimó un promedio anual, para Bogotá y para las nueve zonas en que se divide (gráfica 13); las pruebas estadísticas se hicieron con estas nuevas series.

De acuerdo con la metodología indicada, el primer paso es hacer la prueba global (BASDF) para identificar múltiples burbujas. El cuadro 2 muestra los resultados para Bogotá y para las nueve zonas. Como se puede observar, se encontró evidencia de que en todas las zonas hay múltiples periodos de crecimiento explosivo. Después de presentar los resultados de la prueba global, la gráfica 14 presenta los resultados de las pruebas realizadas para identificar desde cuándo hay crecimiento explosivo en cada zona. En estas se ilustra que el comportamiento de las series, es explosivo cuando supera los valores críticos al 90 y al 95\% (líneas horizontales). 


\section{Gráfica13}

Índice promedio anual real por zona, diciembre de 2002-mayo de 2014

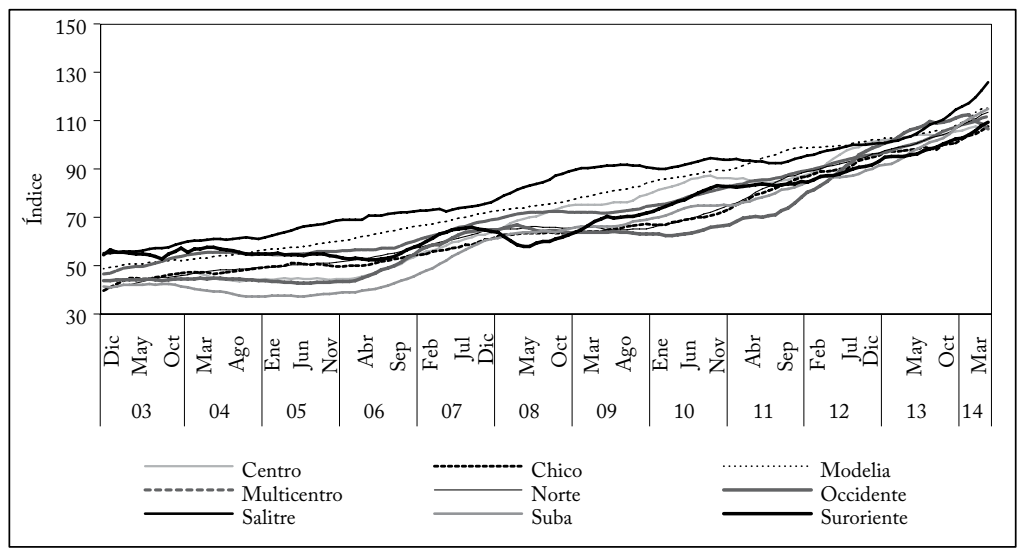

Fuente: La Galería Inmobiliaria, cálculos propios.

Cuadro 2

Valores críticos y estadísticos BASDF de las series, diciembre de 2002-mayo de 2014 (Promedio anual del IPVNG)

\begin{tabular}{lclrrrr}
\hline \multirow{2}{*}{$\begin{array}{c}\text { Número } \\
\text { de datos }\end{array}$} & \multirow{2}{*}{ Ventana } & Índice zona & \multirow{2}{*}{ BASDF } & \multicolumn{3}{c}{ Valores críticos } \\
\hline 124 & 12 & Centro & 6,52 & $90 \%$ & $95 \%$ & $99 \%$ \\
127 & 12 & Chicó & 8,81 & 2,47 & 2,87 & 4,14 \\
127 & 12 & Modelia & 6,02 & 2,47 & 2,82 & 4,14 \\
127 & 12 & Multicentro & 7,92 & 2,47 & 2,82 & 4,14 \\
127 & 12 & Norte & 9,80 & 2,47 & 2,82 & 4,14 \\
127 & 12 & Occidente & 10,64 & 2,47 & 2,82 & 4,14 \\
127 & 12 & Salitre & 11,25 & 2,47 & 2,82 & 4,14 \\
127 & 12 & Suba & 13,02 & 2,47 & 2,82 & 4,14 \\
127 & 12 & Suroriente & 4,94 & 2,47 & 2,82 & 4,14 \\
\hline
\end{tabular}

Fuente: IPVNG: La Galería Inmobiliaria, cálculos propios.

Los resultados de las pruebas univariadas indican que hay un crecimiento explosivo en varias zonas y en diferentes periodos. En primer lugar se identifica claramente un grupo de zonas donde el aumento de precios es persistente después de la recuperación de comienzos de la década. En este grupo, que incluye las zonas de Multicentro, Chicó y Norte, los precios tienen un comportamiento similar: un crecimiento exuberante desde 2006, una atenuación de ese incremento debida a la desaceleración económica de finales de 2008, para luego experimentar crecimientos exuberantes, hasta tal punto que son los más marcados de la ciudad. 


\section{Gráfica 14}

\section{Centro}

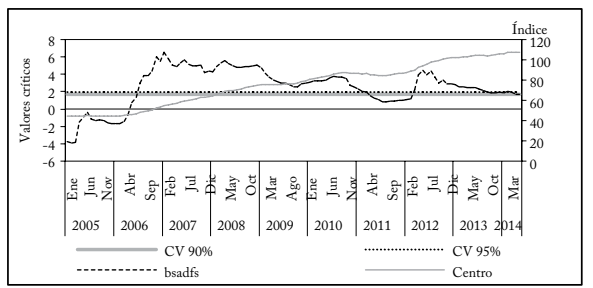

\section{Modelia}

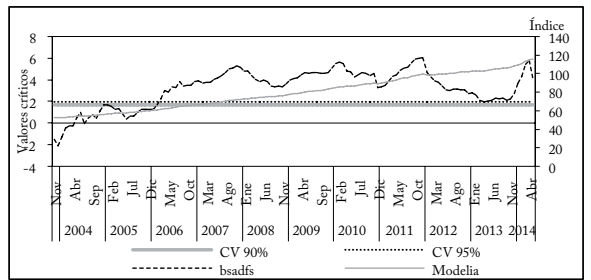

\section{Norte}

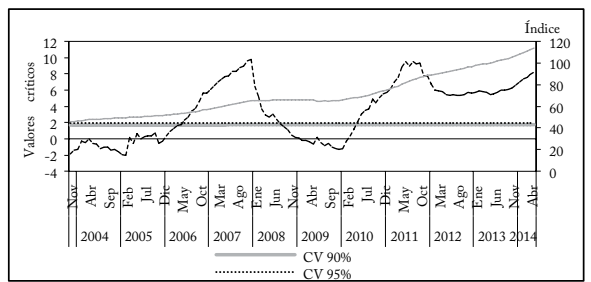

\section{Salitre}

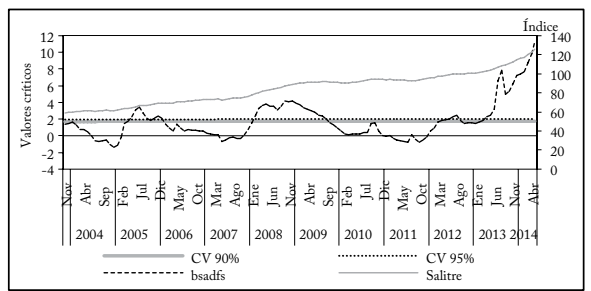

\section{Suroriente}

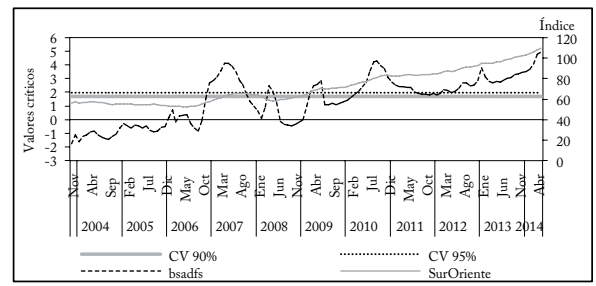

Fuente: precios: La Galería Inmobiliaria, ICCV: Dane; cálculos propios.

\section{Chicó}

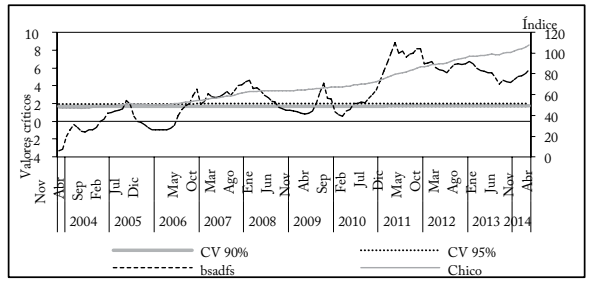

\section{Multicentro}

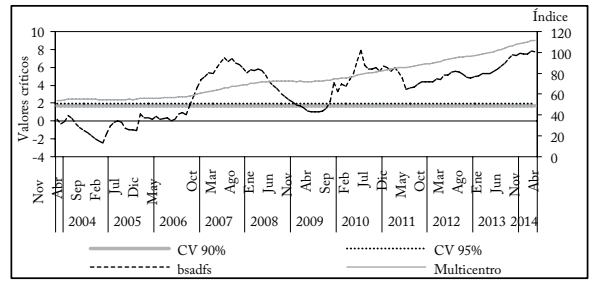

\section{Occidente}

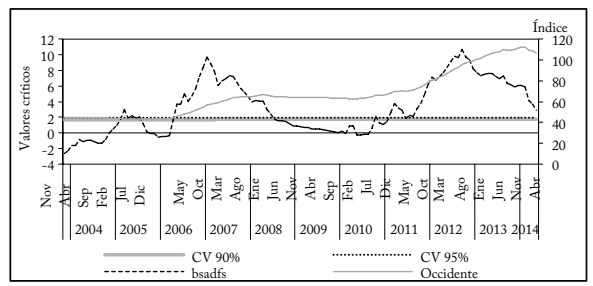

\section{Suba}

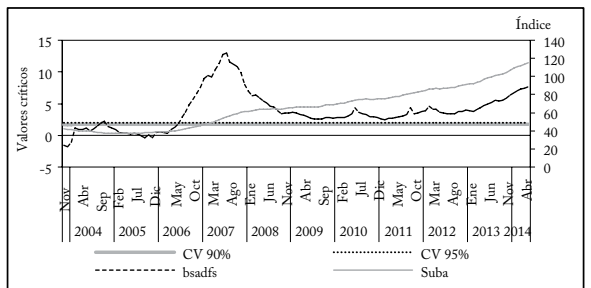


En las demás zonas también se presentan crecimientos explosivos, pero con otra periodicidad y debido a otros factores. En las zonas donde predominan los estratos medios, como Modelia, Salitre y Suba, el crecimiento explosivo se atenúa con el tiempo, especialmente en Salitre, pero los precios vuelven a aumentar en forma exuberante desde 2013. En cambio, en las zonas Centro y Occidente, el comportamiento de los precios deja de ser explosivo en 2014. En la zona Suroriental, donde el niveles de precios de la vivienda es menor, muestra un comportamiento similar al de las zonas de estratos medios, pues el crecimientos de los precios empieza a ser exuberante desde 2013.

\section{CONCLUSIONES}

Para determinar qué está sucediendo con los precios de la vivienda en Bogotá, este artículo primero analiza algunos hechos estilizados y luego emplea el método econométrico univariado para establecer si los precios de la vivienda muestran un comportamiento de burbuja. Este análisis se hizo para Bogotá y nueve zonas de la ciudad, con datos de precios del periodo enero 2002-mayo 2014. Los resultados proporcionan evidencia estadística de crecimientos exuberantes de precios en algunas zonas desde 2009, y además que los incrementos no son homogéneos en la ciudad ni coyunturales.

En las zonas de Chicó, Multicentro y Norte, donde habitan los hogares de ingresos más altos, se detectan los crecimientos explosivos más pronunciados. En este segmento del mercado inmobiliario se observan, como es de esperar, los precios por metro cuadrado más altos de la ciudad, lo que incentiva a las empresas a concentrar la construcción de nuevas vivienda en esas zonas. $\mathrm{El}$ análisis de los hechos estilizados indica que hay sobreoferta de vivienda en los estratos 4, 5 y 6 , y que las ventas superan las necesidades de los hogares respectivos (no hay déficit cuantitativo de vivienda en estos estratos); por su parte, el indicador de rotación ha aumentado en los últimos tiempos (SDP, 2014). Esta constatación parece confirmar que en Chicó, Multicentro y Norte hay inversionistas que pueden estar especulando con los precios de la vivienda, pues la inversión en vivienda no parece estar dirigida principalmente al uso habitacional sino a la obtención de ganancias mediante la valorización, dadas las bajas tasas de interés en el mundo y las expectativas de que los precios continúen aumentando.

En algunas zonas de estratos medios y bajos -Salitre, Modelia, Suba y Suroriente- también se detectan crecimientos explosivos, especialmente a partir de 2013. Pero este comportamiento de los precios 
debe ser explicado por razones diferentes al de las anteriores, como bien muestran los indicadores de déficit habitacional y de rotación.

En el caso de los estratos 1, 2 y 3 (e incluso del estrato 4, salvo en el periodo más reciente) se constata que hay déficit de vivienda, pues la formación de nuevos hogares es bastante superior a la oferta de vivienda nueva, lo que no solo no satisface la necesidad de vivienda de estos hogares sino que amplía el déficit habitacional; esto significa que el incremento de los precios de la vivienda en las zonas donde predominan estos estratos está más relacionada con la presión de la demanda de los hogares que han mejorado su ingreso y con programas del gobierno, como el FRECH, (Piraquive y Hernández, 2014), que con la acción de agentes especuladores.

En el artículo se muestra que la formación de una burbuja puede estar inducida por diversos factores y que no depende de la forma de financiación; aunque esta sí influye en la gravedad de los efectos de la explosión de la burbuja. En general, cuando el crecimiento exuberante de los precios es impulsado por demandantes que compran con recursos de crédito, las consecuencias de dicha explosión suelen ser más graves que cuando compran con recursos propios. Esto último es lo que está sucediendo en Bogotá, pues en promedio los hogares hoy solicitan al sector financiero menos del $50 \%$ de la suma que necesitan para comprar vivienda; se debe añadir, además, la buena calidad de los indicadores de la cartera hipotecaria, así como los bajos niveles cartera/PIB en comparación con los que se observaron durante la crisis de 1998. Estos hechos sugerirían que de haber una corrección a la baja en los precios de la vivienda, los efectos sobre la economía no serían tan graves como en 1998. Pero aun si ese fuese el caso sigue siendo necesario el seguimiento continuo del comportamiento de los precios de la vivienda, del crecimiento y de los cambios en la cartera hipotecaria y de la economía en su conjunto, para advertir a tiempo los efectos del posible ajuste de los precios de la vivienda y atenuar los perjuicios que pueda ocasionar a los hogares y al mismo sector de la construcción. 


\section{AneXo}

Evolución de las ventas de vivienda usada

Acumulado doce meses, enero de 2003-agosto de 2014

Estrato 4

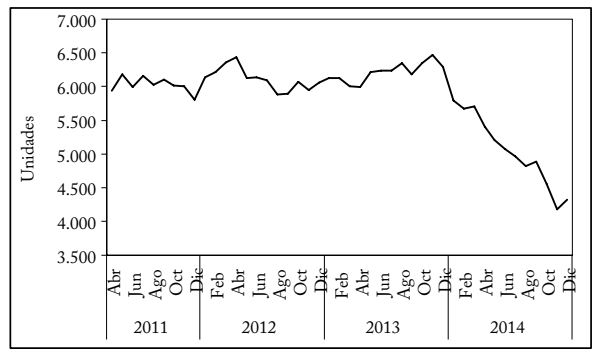

Estrato 5

\section{Estrato 6}
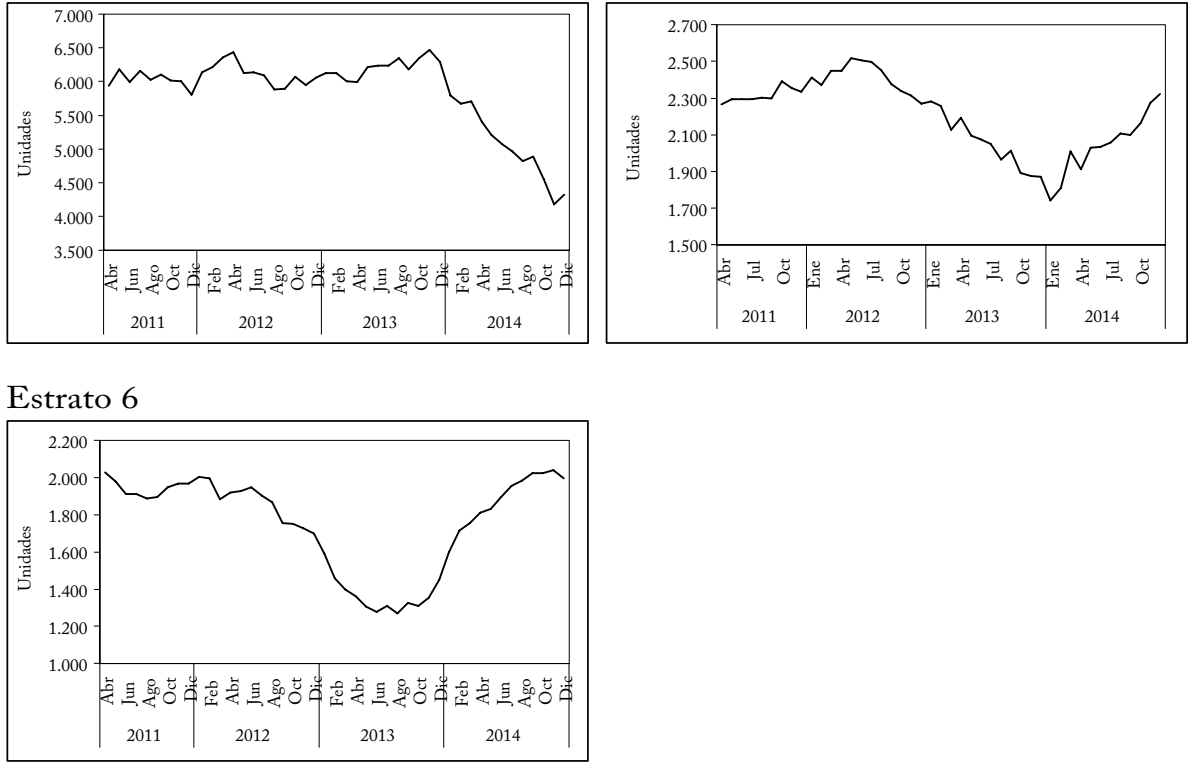

Fuente: La Galería Inmobiliaria, cálculos propios.

\section{REFERENCIAS BIBLIOGRÁFICAS}

1. Blanchard, O y D. Enrri. Macroeconomía: teoría y política económica con aplicaciones a América Latina, Buenos Aires, Prentice-Hall, 2000.

2. Clavijo, S.; M. Janna y S. Muñoz. "La vivienda en Colombia: sus determinantes socioeconómicos y financieros", Borradores de Economía 300, 2004.

3. Crowe, C.; G. Dell'Ariccia et al. "How to deal with real estate booms: Lessons from country experiences", IMF working paper 2011, pp. 1-58.

4. Cuervo, N. y S. Jaramillo. "Dos décadas de política de vivienda en Bogotá apostando por el mercado”, Documentos CEDE 31, 2009.

5. Banco de la República. Reporte de estabilidad financiera abril 2013, Bogotá, BR, 2013.

6. Lonja de Bogotá. Estudio del valor del suelo urbano en Bogotá, Bogotá, 2012 .

7. Gomez, J.; J. Ojeda et al. "Testing for bubbles in housing markets: New results using a new method", Borradores de Economia 753, 2013.

8. Hofstetter, M.; J. Tovar y M. Urrutia. "Effects of a mortgage interest rate subsidy: Evidence from Colombia”, Bogotá, CEDE, 2011.

9. Jaramillo, S y N. Cuervo B. "Precios inmobiliarios de vivienda en Bogotá 1970-2013”, Documentos CEDE 18, 2014. 
10. Joshi, H. "Identifying asset price bubbles in the housing market in India. Preliminary evidence", Reserve Bank of India Occasional Papers 27, 1-2, 2006, pp. 73-88.

11. López, E. y A. Salamanca. "El efecto riqueza de la vivienda en Colombia”, Borradores de Economia 551, 2009.

12. McCarthy, J. y R. W. Peach. "Are home prices the next 'bubble'?", FRBNY Economic Policy Review 10, 3, 2004, pp. 1-17.

13. Phillips, P. C.; Y. Wu y J. Yu. "Explosive behavior in the 1990s Nasdaq: When did exuberance escalate asset values?", International Economic Review 52, 1, 2011, pp. 201-226.

14. Phillips, P. C.; S. Shi y J. Yu. “Testing for multiple bubbles”, New Haven, Cowles Foundation discussion paper, 2012.

15. Piraquive, G. y G. Hernández. "Evolución de los precios de la vivienda en Colombia”, Bogotá, DNP, 2014.

16. Rosser, J. B.; M. V. Rosser y M. Gallegati. "A Minsky-Kindleberger perspective on the financial crisis", Journal of Economic Issues 46, 2, 2012, pp. 449-458.

17. Salazar, N.; R. Steiner et al. “¿Qué tan desalineados están los precios de la vivienda en Colombia?”, Bogotá, Fedesarrollo, 2012.

18. Secretaría Distrital de Planeación. “¿Burbuja inmobiliaria en Bogotá?”, Observatorio Dinámicas del Territorio, SDP, Boletín No. 12, 2013a.

19. Secretaría Distrital de Planeación. "Burbuja inmobiliaria en Bogotá", Observatorio Dinámicas del Territorio, SDP, Boletín No. 12, 2103b.

20. Secretaría Distrital de Planeación. "Determinantes de la tenencia de vivienda en Bogotá”, Observatorio Dinámicas del Territorio, SDP, Boletín No. 17, 2014a.

21. Secretaría Distrital de Planeación. "Seguimiento mercado de vivienda”, Observatorio Dinámicas del Territorio, SDP, Boletín No. 18, 2014b.

22. Shiller, R. J. Irrational exuberance, Random House Digital, 2005.

23. Smith, M. H. y G. Smith. "Bubble, bubble, where's the housing bubble?", Brookings Papers on Economic Activity, 2006, 1, pp. 1-67. 